\title{
Bioinformatics Analysis of the Z-Carotene Desaturase Gene in Potato (Solanum Tuberosum)
}

\author{
Jie Ma ${ }^{1,2}$, Ping Zhou ${ }^{1,2}$, Chunyan Chen ${ }^{2}$, Min Jiang ${ }^{1}$, Meiling $\mathrm{Wu}^{2}$, Rui $\mathrm{Wu}^{2}$, \\ Chaohai Wang ${ }^{2}$ and Bo Sun ${ }^{1, a, ~ *}$ \\ ${ }^{1}$ College of Horticulture, Sichuan Agricultural University, Chengdu, China \\ ${ }^{2}$ Bijie Institute of Agricultural Science, Bijie, Guizhou, China \\ a14099@sicau.edu.cn.
}

Keywords: Solanum lycopersicum; Z-carotene desaturase; Subcellular localization predicted.

\begin{abstract}
Z-carotene desaturase (ZDS) is an important enzyme in carotenoid biosynthesis. Here, the Solanum tuberosum ZDS (StZDS) gene sequences were obtained from Spud DB database, and preformed for bioinformatics analysis. The StZDS gene mapped to chromosomes 1, and contains an open reading frame of $1,767 \mathrm{bp}$ that encodes a 588 -amino acid protein with a calculated molecular mass of $64.70 \mathrm{kD}$ and an isoelectric point (pI) of 8.6. Subcellular localization predicted the StZDS gene was in the chloroplast. The conserved domain of the StZDS protein is PLN02487. The ZDS protein is most closely related to Solanum lycopersicum. The findings of the present study provide a molecular basis for the elucidation of ZDS gene function in potato.
\end{abstract}

\section{Introduction}

Potato (Solanum tuberosum) is ranked as the third most important food crop in the world. Potato is not only of importance as a food crop, and also one of the major crops grown for starch production [1]. It yields a high-energy output per hectare, and is a rich source of nutrients, including carbohydrates, and carotenoids [1-2].

Carotenoids, which are synthesized in various photosynthetic and non-photosynthetic organisms, including algae, plants, and some bacteria and fungi, are a class of 40-carbon hydrocarbon compounds derived from a terpenoid precursor [3-5]. Carotenoids represent a diverse group of pigments that contribute to the red, orange, and yellow colors of various horticultural plants, contributing to their economic value [5-6]. Carotenoids participate in various plant physiological processes, including growth, development, and responses to multiple environmental factors. In green tissues, these act as accessory pigments for the assembly of photosystems and light-harvesting antenna complexes and also have photoprotective functions during photosynthesis [3,5]. In non-green tissues, these confer distinct colors to specialized plastids known as chromoplasts, which contribute to the sundry colors in plants $[4,6]$. In addition, carotenoids are the precursors of numerous volatile flavor compounds and phytohormones such as abscisic acid (ABA) and strigolactones [4]. Carotenoids are also essential compounds of human diets, primarily as a precursor of vitamin A [7]. Moreover, carotenoid intake could also reduce the risk for cancer and cardiovascular disease [8-9].

ZDS is an important enzyme in carotenoid biosynthesis, catalyzing the 9,9'-di-cis- $\zeta$-carotene into 7,9,7',9'-tetra-cis-lycopene [5]. The genes encoding the ZDS protein have been isolated in various plant species, including Arabidopsis [10], N. tabacum [11], tomato [12], and ornamental sunflower [13]. To date, research studies on ZDS in potato are limited. In the present study, the ZDS gene sequence of potato was obtained from web database, and then bioinformatics analysis of the ZDS gene was analyzed. The present study aimed to establish the foundation for further studies on the molecular mechanism of ZDS in potato. 


\section{Materials and Methods}

\subsection{Sequence Obtention of the StZDS Gene}

The genomic DNA and mRNA sequences of ZDS gene of potato were downloaded and obtained from The Spud DB database (http://solanaceae.plantbiology.msu.edu), and then used to subsequent bioinformatic analysis.

\subsection{Bioinformatics Analysis of the StZDS Gene}

The amino acid sequence, protein molecular weight, isoelectric point, stability index, and hydrophobicity of the StZDS gene were analyzed and predicted by ExPASy (http://web.expasy.org) and NCBI (https://www.ncbi.nlm.nih.gov/). Subcellular localization was predicted by WoLF PSORT (http://www.genscript.com/wolf-psort.html). The conserved domain were predicted by NCBI (https://www.ncbi.nlm.nih.gov/Structure/cdd/wrpsb.cgi). Phylogenetic tree analysis of the ZDS proteins was executed in MEGA 6.0 using the neighbor-joining (NJ) method.

\section{Results}

\subsection{Analysis on Genomic Organization}

The Spud DB database was used to analyze the chromosomal localization and genomic organization of StZDS. The gene ID in Spud DB database is PGSC0003DMT400057865. The StZDS gene was mapped to chromosomes 1 and has 14 exons and 13 introns (Fig. 1).

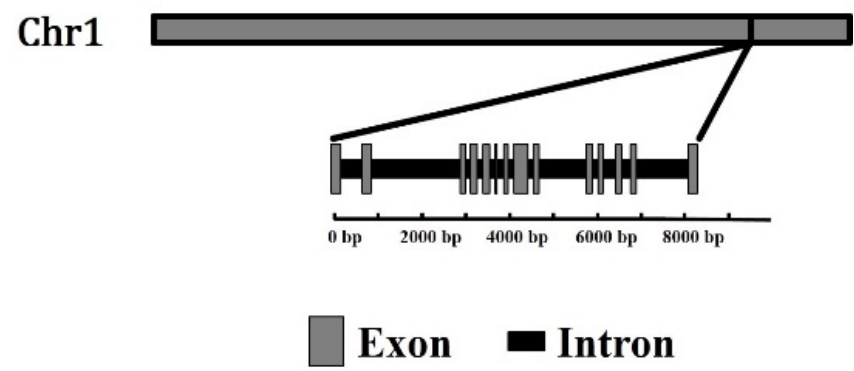

Fig. 1 Chromosomal location and genomic structure of StZDS.

\subsection{Protein Physical and Chemical Properties Analysis}

Sequence analysis indicated that the StZDS gene contained a 1,767-bp open reading frame (ORF), which encoded a 588-amino acids protein with a calculated molecular mass of $64.70 \mathrm{kD}$ and an isoelectric point (pI) of 8.6. The amino acid types and proportions of the StZDS gene was shown in Figure 2, the highest number of amino acid is Leucine (Leu), whereas the lowest number is Tryptophan (Trp). Its predicted formula was $\mathrm{C}_{2902} \mathrm{H}_{4579} \mathrm{~N}_{781} \mathrm{O}_{845} \mathrm{~S}_{24}$. Its total average hydrophilicity index was -0.104 , liposoluble index was 89.71 , and instability index in solution was 37.36.

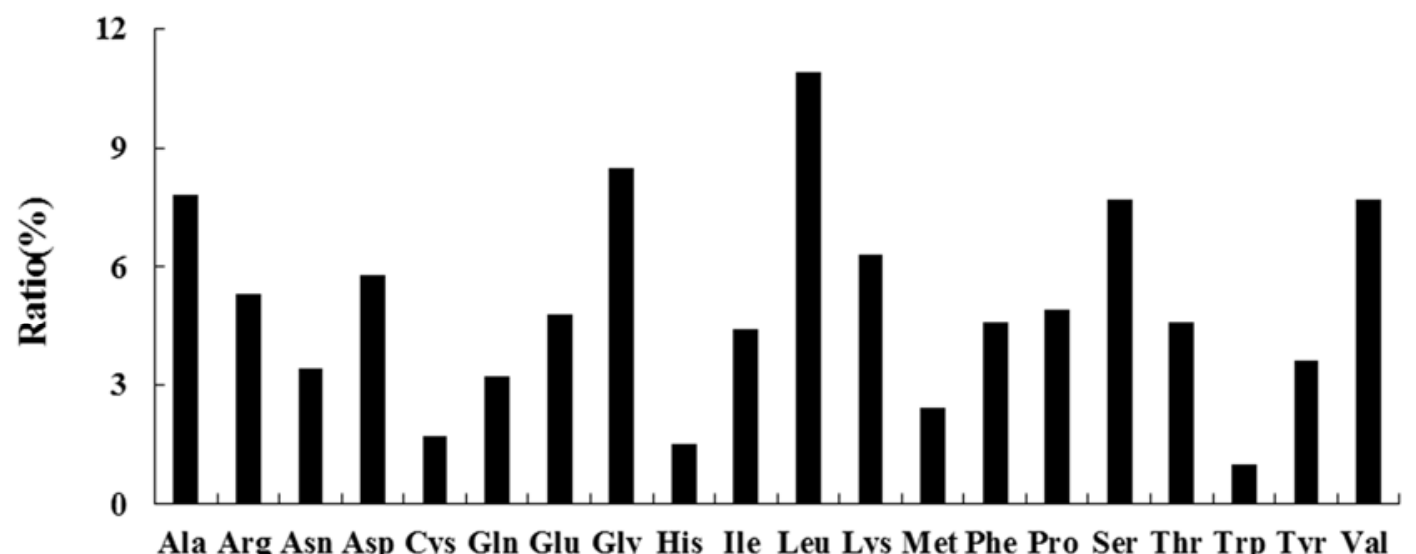

Amino Acid

Fig. 2 Amino acid composition of StZDS 


\subsection{Subcellular Localization and Conserved Domain Analysis}

Subcellular localization of the StZDS gene was predicted by WoLF PSORT to be in the chloroplast. The analysis using Conserved Domain Database (CDD) demonstrated that the amino acid sequence of the StZDS protein has one conserved domain PLN02487 and one superfamily COG2907 with predicted NAD/FAD-binding protein structure.

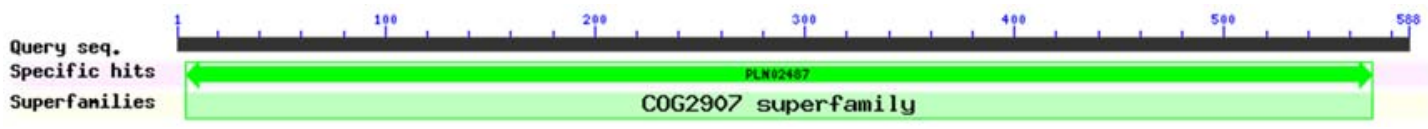

Fig. 3 Conserved domains analysis of StZDS

\subsection{Homology and Phylogenetic Tree Analysis}

A phylogenetic tree was constructed to illustrate the relationship among the ZDS proteins of potato and 19 other higher plant species (Fig. 4). A total of two major clusters were identified, one cluster includes Solanaceae and Rutaceae, while the other cluster includes Malvaceae and Rosaceae. Sequence alignment indicated that the StZDS protein is more closely related to Solanum lycopersicum, which belonged to the Solanaceae branch.

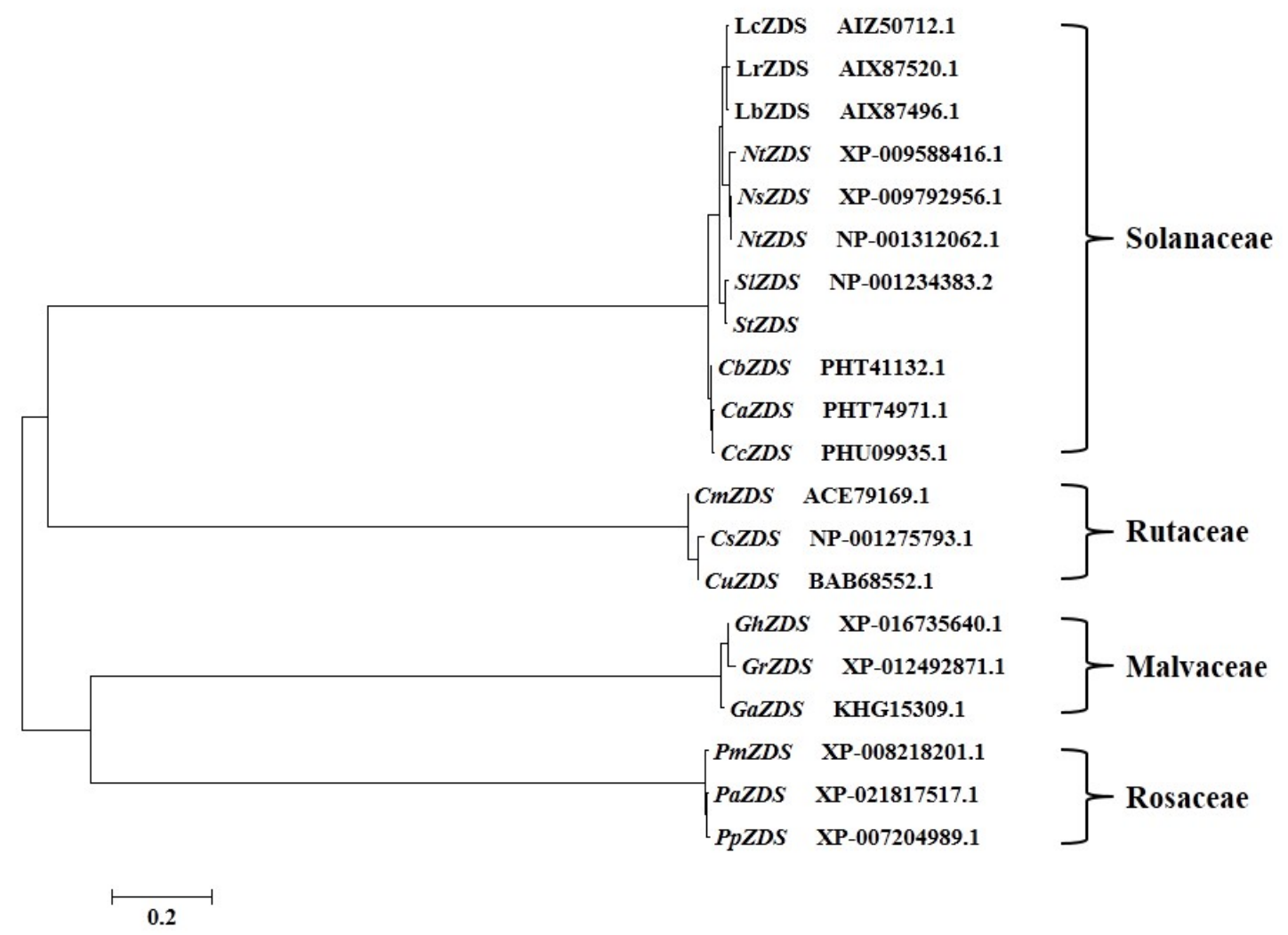

Fig. 4 Phylogenetic tree analysis of StZDS and ZDS proteins of other species

\section{Discussion}

The present study analyzed the StZDS gene of potato. ZDS enzyme is encoded by single-copy gene in tomato, grape, and Arabidopsis [5-6]. Moreover, ZDS gene occurred as a single copy in potato, indicating that the enzyme may have undergone similar evolutionary patterns. Previous studies have shown that the ZDS protein is relatively conserved in plants $[11,13]$. The ZDS protein of Helianthus annuus is similar to the ZDS protein of Tagetes erecta and Chrysanthemum $\times$ morifolium, showing $96.4 \%$ and $90.1 \%$ homology [13]. The findings of the present study show that ZDS from potato is highly conserved in plants, similar to that observed in earlier reports. The findings of the present study may serve as a foundation for future studies on the functions of ZDS in carotenoid metabolism in potato. 


\section{Acknowledgements}

Correspondence author: Bo Sun, Tel: +8628 86291941, E-mail addresses: 14099@sicau.edu.cn. This work was supported by Science and Technology Foundation of Guizhou Province (QianKeHeJiChu [2016]1003), Talent development project of Bijie City (BiKeHe(2015)37), Science and Technology Achievement Transformation and Guidance Fund Project of Guizhou Province (QianKeHeChengZhuanZi[2014]5025), and Science and Technology Special Fund Project of Central Subsidized Place (QianKeHeTiaoZhongBuDi[2015]4003).

\section{References}

[1]. Andersson, M., H. Turesson, A. Nicolia, A. Fält, M. Samuelsson, and P. Hofvander. 2017. "Efficient targeted multiallelic mutagenesis in tetraploid potato (Solanum tuberosum) by transient CRISPR-Cas9 expression in protoplasts," Plant Cell Rep., 36: 117-128.

[2]. Lu, S., J. Van Eck, X. J. Zhou, A. B. Lopez, D. M. O’Halloran, K. M. Cosman, B. J. Conlin, D. J. Paolillo, D. F. Garvin, J. Vrebalov, L. V. Kochian, H. Küpper, E. D. Earle, J. Cao, L. Li. 2006. "The cauliflower Or gene encodes a dnaJ cysteine-rich domain-containing protein that mediates high levels of beta-carotene accumulation," Plant Cell, 18: 3594-3605.

[3]. Pizarro, L., and C. Stange. 2009. "Light-dependent regulation of carotenoid biosynthesis in plants,” Cien. Inv. Agr., 36: 143-162.

[4]. Liu, L. H., Z. Y. Shao, M. Zhang, and Q. M. Wang. 2015. "Regulation of carotenoid metabolism in tomato," Mol. Plant, 8: 28-39.

[5]. Nisar, N., L. Li, S. Lu, N. C. Khin, and B. J. Pogson. 2015. "Carotenoid metabolism in plants," Mol. Plant, 8: 68-82.

[6]. Tuan, P.A., J. K. Kim, J. Lee, W. T. Park, D. Y. Kwon, Y. B. Kim, H. H. Kim, H. R. Kim, and S. U. Park. 2012. "Analysis of carotenoid accumulation and expression of carotenoid biosynthesis genes in different organs of Chinese cabbage (Brassica rapa subsp. pekinensis)," Excli J., 11: 508-516.

[7]. Krinsky, N. I., and E. J. Johnson. 2005. "Carotenoid actions and their relation to health and disease," Mol. Aspects. Med., 26: 459-516.

[8]. Mayne, S. T. 1996. "Beta-carotene, carotenoids, and disease prevention in humans," FASEB J., 10: 690-701.

[9]. Giovannucci, F. 1999. "Tomatoes, tomato-based products, lycopene, and cancer: review of the epidemiologic literature,” J. Natl. Cancer Inst., 91: 317-331.

[10]. Scolnik, P. A., and G. E. Bartley. 1995. "Nucleotide sequence of zeta-carotene desaturase (accession no. U38550) from Arabidopsis," Plant Physiol., 109: 1499.

[11]. Shi, Y. M., P. Wei, Y. Y. Chen, R. Yang, L. F. Jin, P. P. Liu, F. Li, L. B. Qu, F.C. Lin, and R. Wang. 2015. "Cloning and functional analysis of $\zeta$-carotene desaturase gene in Nicotiana tabacum," Tobacco Sci. \& Technol., 48: 1-8.

[12]. Giuliano, G., G. E. Bartley, and P. A. Scolnik. 1993. "Regulation of carotenoid biosynthesis during tomato development," Plant Cell, 5: 379-387.

[13]. Zhong, H. Z., J. S. Wu, M. L. Huang, R. H. Fan, Y. H. Luo, B. Lin, and X. X. Ye. 2015. "Cloning and expression of $\zeta$-carotene desatura gene in ornamental sunflower," J. Plant Genetic Resources, 16: 828-835. 